\title{
Research Square \\ Enhancing Positron Production using Front Surface Target Structures
}

\section{Sheng Jiang ( $\nabla_{\text {jiang8@llnl.gov ) }}$}

Lawrence Livermore National Laboratory https://orcid.org/0000-0002-0381-3846

\section{Anthony Link}

LLNL

\section{Dave Canning}

Laboratory For Laser Energetics

Julie Fooks

General Atomics

\section{Paul Kempler}

California Institute of Technology

\section{Shaun Kerr}

Lawrence Livermore National Laboratory

J Kim

University of California, San Diego

\section{Michael Krieger}

Laboratory For Laser Energetics

\section{Nathan Lewis}

California Institute of Technology

\section{Russell Wallace}

LLNL

\section{G. Jackson Williams}

Lawrence Livermore National Laboratory https://orcid.org/0000-0002-6495-5696

\section{Sisir Yalamanchili}

California Institute of Technology

Hui Chen

Lawrence Livermore National Laboratory

\section{Article}

Keywords: positrons, laser, plasma, front-surface target structures

Posted Date: August 21st, 2020 
DOI: https://doi.org/10.21203/rs.3.rs-53834/v1

License: (c) (1) This work is licensed under a Creative Commons Attribution 4.0 International License. Read Full License 


\section{Enhancing Positron Production using Front Surface Tar- get Structures}

3 S. Jiang ${ }^{1}$, A. Link ${ }^{1}$, D. Canning ${ }^{2}$, J. A. Fooks ${ }^{3}$, P. A. Kempler ${ }^{4}$, S. Kerr ${ }^{1}$, J. Kim ${ }^{5}$, M. Krieger ${ }^{2}$, N.

${ }_{4}$ S. Lewis $^{4}$, R. Wallace ${ }^{1}$, G. J. Williams ${ }^{1}$, S. Yalamanchili ${ }^{4} \&$ H. Chen ${ }^{1}$

$5{ }^{1}$ Lawrence Livermore National Laboratory, Livermore, California, USA

$6 \quad{ }^{2}$ Laboratory For Laser Energetics, Rochester, New York, USA

$7{ }^{3}$ General Atomics, San Diego, California, USA

$8{ }^{4}$ California Institute of Technology, Pasadena, California, USA

$9{ }^{5}$ Center for Energy Research, University of California San Diego, San Diego, California, USA

We report the first experimental results and simulations that demonstrate a substantial effect of large-scale front-surface target structures on high-intensity laser-produced positrons. Specifically, as compared to a flat target under nominally the same laser conditions, an optimized Si microwire array target yielded a near $100 \%$ increase in the laser-to-positron conversion efficiency and produced a $10 \mathrm{MeV}$ increase in positron energy. Full-scale particle-in-cell simulations that modeled the entire positron production and transport process starting from laser-plasma interactions provided additional insight into the beneficial role of target structuring. The agreement between experimental and simulated spectra suggests future target structure optimization for desired positron sources.

Electron-positron pair plasmas are found in various extreme astrophysical objects, such as pulsars, bipolar outflows, active galactic nuclei, and gamma ray bursts ${ }^{1}$. Producing a pair plasma 
with similar conditions in the laboratory is extremely challenging but could significantly deepen the understanding of these exotic objects ${ }^{2-5}$. With the advances in high intensity laser technology, several methods for pair production have been either demonstrated or proposed, with different mechanisms dominating the physics in different regimes of laser intensity. For example, the Schwinger mechanism ${ }^{6}$ requires an extremely high intensity, above $\sim 10^{29} \mathrm{~W} / \mathrm{cm}^{2}$, for spontaneous pair creation from vacuum, whereas the Breit-Wheeler (BW) mechanism ${ }^{7}$ requires about $10^{25} \mathrm{~W} / \mathrm{cm}^{2}$ for avalanche-type discharge ${ }^{8}$. These intensities are far beyond the capability of state-of-the-art lasers (up to $10^{22} \mathrm{~W} / \mathrm{cm}^{2}$ ).

An alternative method is to inject laser produced high-energy electrons into high- $\mathrm{Z}$ target materials ${ }^{4,9-15}$, with the electrostatic field of the nucleus involved in the pair production process releasing the constraint on the laser E field intensity. As these high-energy electrons transport through the material, positrons are produced via two major mechanisms: the trident process and the Bethe-Heitler $(\mathrm{BH})$ process ${ }^{16}$. The latter process dominates when a thick target is used. In a laser experiment, pair production via the $\mathrm{BH}$ process includes three steps. First, relativistic electrons are generated through a laser plasma interaction (LPI) at the front side of the target. These electrons then transport through the high- $\mathrm{Z}$ material and produce high-energy photons via Bremsstrahlung radiation. Retardation of the high-energy photons in the field of nucleus then creates electron-positron pairs. The key step is to transfer laser energy into enough high-energy (10s of MeV) electrons, for which, only a moderate intensity laser $\left(\sim 10^{20} \mathrm{~W} / \mathrm{cm}^{2}\right)$ is needed. Experiments using this type of setup have produced up to $10^{12}$ pairs/shot, which is the highest yield reported to date by use of lasers. 
Optimizing the positron yield is critical to apply the laser-produced pairs to laboratory astrophysics. Although higher laser intensities or energies can produce a larger pair yield, at present, improvements are needed before lasers can provide enough power to permit scaled laboratory astrophysics experiments.

The electron temperature largely determines the positron yield from the $\mathrm{BH}$ mechanism, so a key to higher positron production is the production of hotter electrons. In addition to increasing the laser intensity, substantial enhancement in electron energies can be obtained by manipulating the laser-plasma interaction using a structured front surface target ${ }^{17,18}$. Specifically, highly-ordered silicon microwire arrays facing the laser pulse enable guiding the relativistic electron beam along the structured surface and moreover facilitiate a direct laser acceleration mechanism. Such an electron beam can then create a substantial enhancement in the Bremstrahlung radiation produced by a high-Z convertor target ${ }^{19}$. The Bremsstrahlung X-rays further interact with atomic nuclei in the convertor target and create more electron-positron pairs through the $\mathrm{BH}$ process.

We demonstrate herein experimentally a substantial enhancement in both the yield and the energy of generated positrons using target structures, which suggests an efficient and inexpensive approach to improvement of positron sources. Particle-in-cell (PIC) simulations with the code Chicago ${ }^{20}$ have been used to explain the experimental results and have allowed a direct simulation of the effects of the laser-plasma interaction (LPI) on the positron yield. Moreover, the simulation is in good qualitative agreement with the experimental data. 


\section{Results}

Experiment. A schematic diagram of the experimental setup is shown in Figure 1(a). The structured target was irradiated with the OMEGA EP laser pulse, with a wavelength of $1.053 \mu \mathrm{m}$, an energy of $500 \mathrm{~J}$, and a pulse length of approximately $700 \mathrm{fs}$. The focal spot at the target was about $30 \mu \mathrm{m}$ in diameter as derived from an on-shot wavefront and far-field measurement. The peak intensity was therefore estimated to be $4.5 \times 10^{20} \mathrm{~W} / \mathrm{cm}^{2}$. Prior to the experiment, the structure geometry (spacing and length) was optimized through PIC simulations of the hot electron temperature. The optimal geometry is an array of silicon microwires with $3 \mu \mathrm{m}$ diameter, $13 \mu \mathrm{m}$ length and $15 \mu \mathrm{m}$ center-to-center transverse distance. For reference, we have also shot flat targets as well as another type of unoptimized control structure that showed detrimental effects on electron energies in simulations. The second type of target had $3 \mu \mathrm{m}$ diameter, $100 \mu \mathrm{m}$ length and $7 \mu \mathrm{m}$ center-to-center transverse distance. The microwires in the latter target have been shown in previous work to be too long in length and too close to each other, so they tend to break the laser pulse and consequently lead to a poor electron spectrum ${ }^{17,18}$.

Figure 1(b) and (c) show scanning electron microscope images of both target structures used in the experiment. The Si microwire arrays $100 \mu \mathrm{m}$ in height were first grown on a $\mathrm{Si}<111>$ wafer by the vapor-liquid-solid growth method ${ }^{21}$, whereas the shorter, optimal microwire arrays were etched from $\mathrm{Si}<100>$ wafers via Deep Reactive Ion Etching ${ }^{22}$. The microwires were then embeded in a $\sim 30 \mu \mathrm{m}$ thick polydimethylsiloxane layer and peeled off of the substrate. This thin polydimethylsiloxane layer was then attached to a $1 \mathrm{~mm}$ thick Au backing layer. In this case, the 


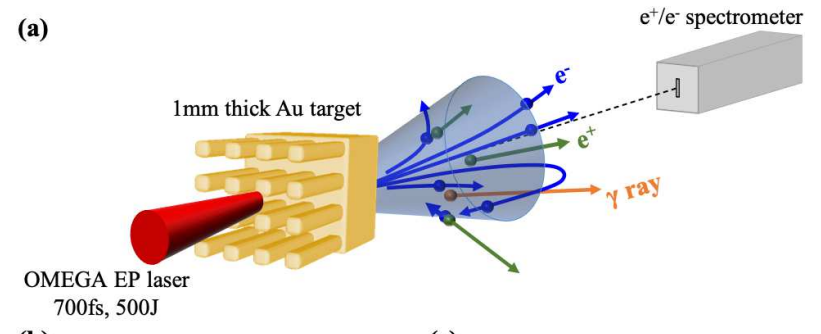

(b)
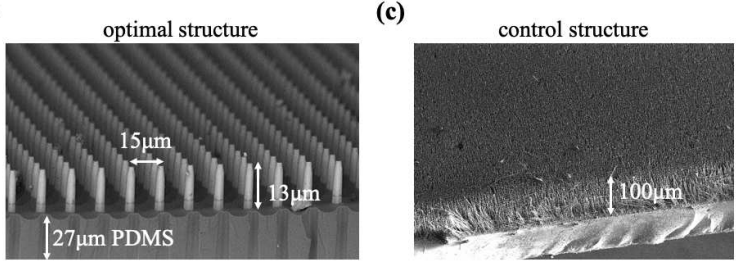

Figure 1: Schematic diagram of the experimental setup and scanning electron microscope (SEM) images of targets. (a) Schematic of the experimental setup. The same setup is used for LPI PIC simulations. The laser has $500 \mathrm{~J}$ energy, $700 \mathrm{fs}$ pulse length and a peak intensity of $4.46 \times 10^{20}$ $\mathrm{W} / \mathrm{cm}^{2}$. Target structures are made of Si wires that reside on a thin piece of polydimethylsiloxane. They are then attached to a $1 \mathrm{~mm}$ thick Au convertor target for positron generation. The electron/positron spectrometer is placed opposite to the laser pulse. (b) SEM image of the optimized target structure. The wires are $3 \mu \mathrm{m}$ in diameter and $15 \mu \mathrm{m}$ apart. They have a total length of 40 $\mu \mathrm{m}$ but their bottom parts are embedded inside a $27 \mu \mathrm{m}$ polydimethylsiloxane layer; therefore the wire structures exposed outside the polydimethylsiloxane is $13 \mu \mathrm{m}$. (c) SEM image of the unoptimized control structure used in the experiment. The Si wire array is about $100 \mu \mathrm{m}$ long (exposed outside polydimethylsiloxane), $3 \mu \mathrm{m}$ in diameter, and $7 \mu \mathrm{m}$ in period. 
high-energy electrons generated and guided by the surface structures would transport through a thick high-Z material ( $\mathrm{Au}$ ) and induce pair production. The transverse size of the Au block used in the experiment was also $1 \mathrm{~mm}$. The laser was directed at normal incidence onto the target and the microwire arrays were oriented along the laser direction. This configuration has been shown in previous work to yield the highest enhancement of electron energy and directionality ${ }^{17,18}$. The positron spectra were measured by an electron/positron spectrometer on the back side of the target along the laser direction (which was also the target normal direction).

(a)

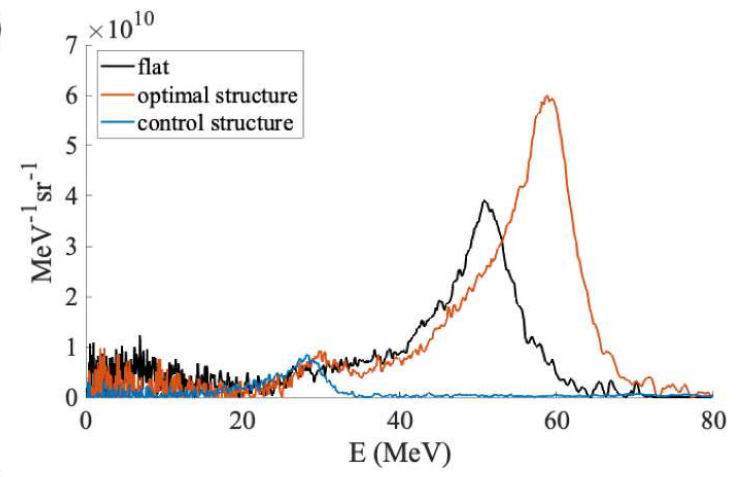

(b)

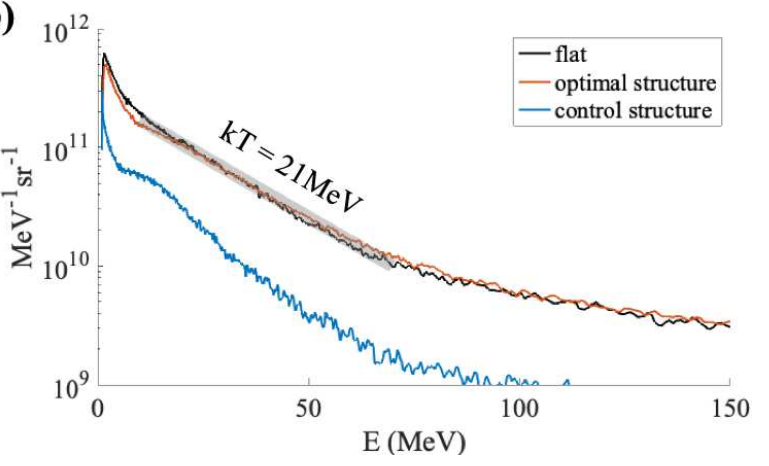

Figure 2: Experimentally measured spectra for (a) positrons and (b) electrons. Different colors indicate the results from different targets under the same laser conditions.

The experimental positron and electron spectra for 3 different types of targets are shown in Figure 2(a) and (b). The optimally structured target generated about 50\% more positrons than the 
regular flat target, and the laser to positron conversion efficiency doubled for the optimal structure compared to the flat subsrate. The spectrum peak also shifted from $\sim 50 \mathrm{MeV}$ for the flat target to $\sim 60 \mathrm{MeV}$ for the optimally structured target. The unoptimized structure showed fewer as well as much lower-energy positrons, in accord with expectations. The electron spectrum from the unoptimized structure also showed the same trend, in agreement with the positron measurements. However, the electron spectra from flat and optimally structured targets were mutually similar, with both having an electron temperature of about $21 \mathrm{MeV}$.

Simulations and Discussions Multiple simulations to model the entire process were performed to elucidate why the measured positron spectrum from optimal structure is obviously superior while its electron spectrum is similar to that from flat target. The simulations used the same laser conditions and target geometries as the experiment. We fitted the measured laser fluence map with two Gaussian functions to maintain the intensity distribution of the experiment. The OMEGA EP laser had a substantial prepulse that could affect the conversion efficiency from the laser to fast electrons, and would therefore affect the yield and energy of positrons. The facility has an on-shot prepulse measurment from 3 ns to $1 \mathrm{~ns}$ prior to the main laser pulse. For the prepulse within $1 \mathrm{~ns}$, we assumed a similar profile to that measured by Dorrer et al. on OMEGA EP ${ }^{23}$. The total energy of the prepulse was about $3.5 \mathrm{~mJ}$. Hydrodynamic simulations with the code Hydra ${ }^{24}$ were used to calculate the preplasma profile, as is shown in Figure 3(a).

Full 3D PIC simulations to model all physics processes are impractical with current supercomputers. We instead adopted a two-stage approach that has been demonstrated on other targets 


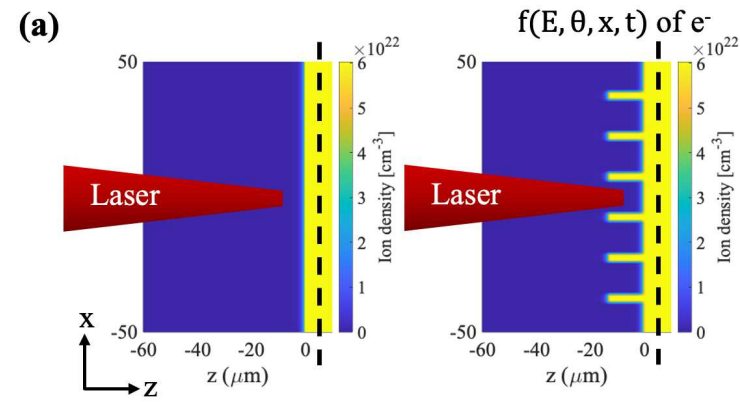

(b)
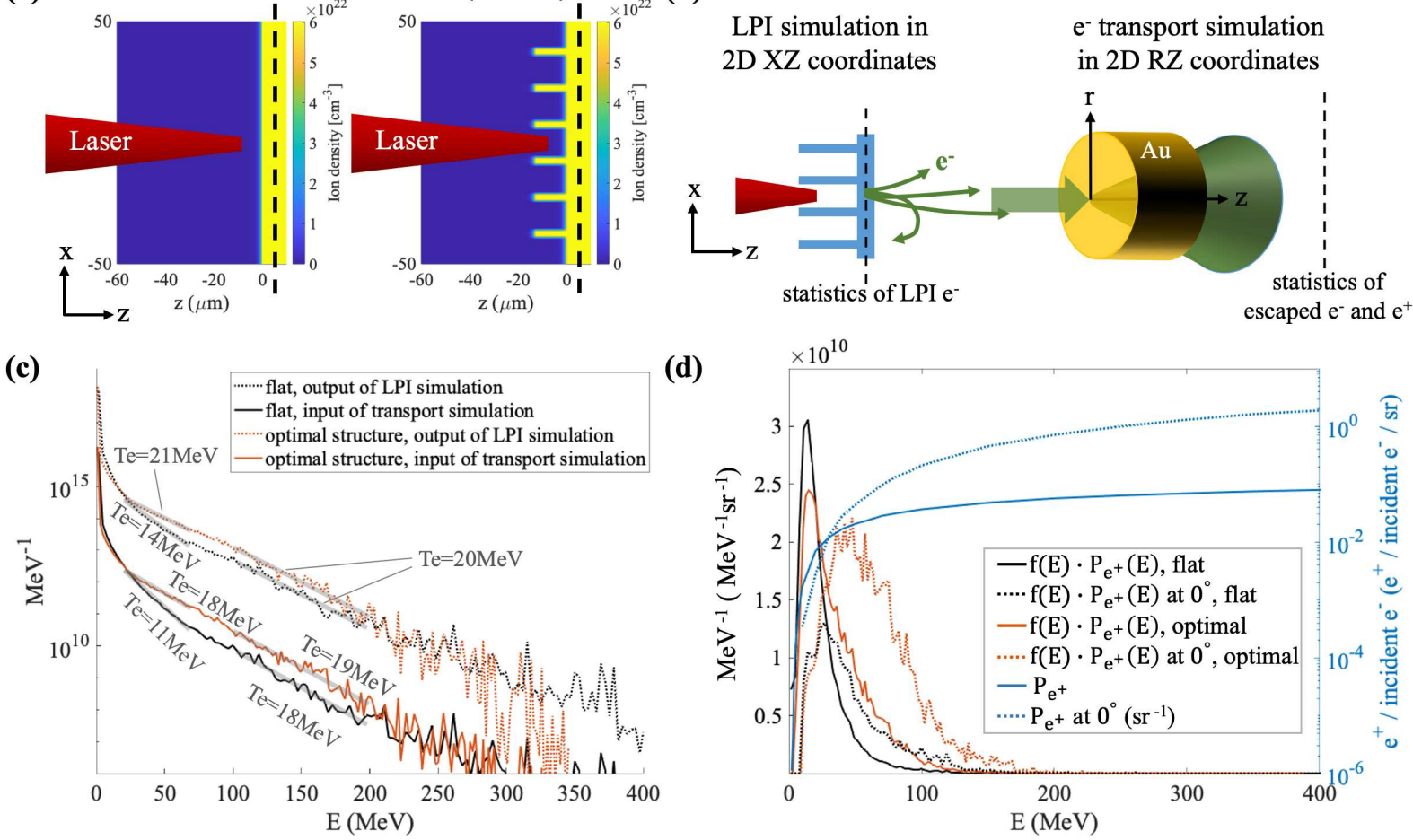

(d)

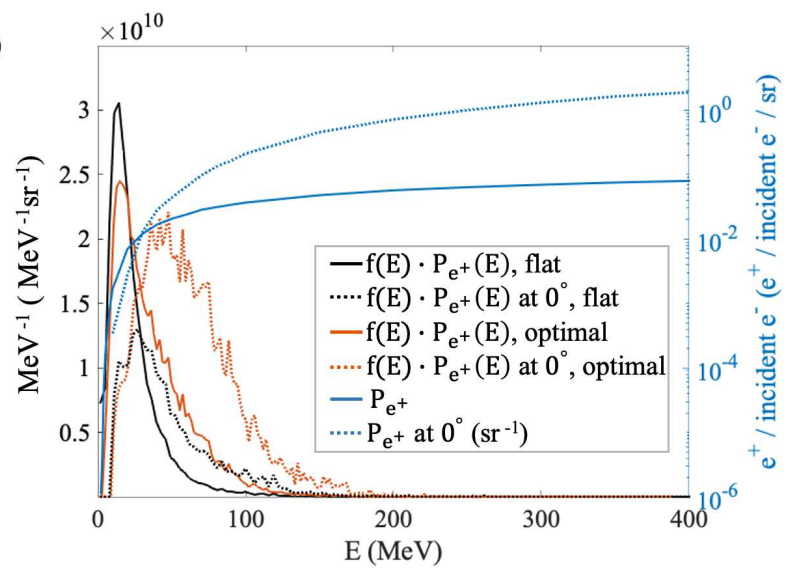

Figure 3: (a) Initial ion density for 2D Cartesian LPI simulations. (b) Schematic diagram of simulation setups. We have injected the fast electrons derived from LPI simulation to the following transport simulation after converting the electron source from Cartesian to cylindrical geometry. (c) Electron spectra inside the target from 2D Cartesian LPI simulations (dashed curves) and spectra of injected electron source for 2D cylindrical transport simulations (solid curves). (d)Solid blue curve (right y axis) shows the probability of one positron generated by one monoenergetic electron transporting through a $1 \mathrm{~mm}$ thick, $1 \mathrm{~mm}$ diameter $\mathrm{Au}$ target, and dashed blue curve shows the probability (per sr) of generating a positron that exits at $0^{\circ}$ with respect to target normal. The black and red curves (with respect to the left y axis) show injection electron spectra multiplied by the positron generation probability as a function of energy. 
25,26 to simulate LPI and transport processes separately. The overall simulation process is illustrated in Figure 3(b). First a 2D Cartesian geometry was used to simulate the LPI process, with only $\mathrm{x}$ and $\mathrm{z}$ dimensions modeled in space. However, the velocity was $3 \mathrm{D}$ as all 3 components $v_{x}$, $v_{y}$ and $v_{z}$ were updated at each time step. We could not use a cylindrical geometry because the laser was linearly polarized in the $\mathrm{x}$ direction. The electrons were measured at a plane that was 5 $\mu \mathrm{m}$ inside the target. The energy, direction, position and time of each electron macroparticle have all been recorded. We then processed the laser-generated electrons to get their distribution $\mathrm{f}(\mathrm{E}, \theta$, $\mathrm{x}, \mathrm{t})$ as a function of energy, angle, transverse distance, and time. Here the angle $\theta$ is defined as $\cos ^{-1}\left(v_{z} / v\right)$. At this point, we assumed a rotational symmetry (in $\phi$ ) along the laser propagation axis for both space and velocity, and converted the distribution to cylindrical coordiates so that $\mathrm{f}(\mathrm{E}$, $\theta, \mathrm{r}, \mathrm{t})=\mathrm{f}(\mathrm{E}, \theta, \mathrm{x}, \mathrm{t})$. The transport simulation was performed in a 2D cylindrical geometry. When hot electrons leave the target, they would create a strong sheath field on the back side. The sheath field can slow down the electrons and cause reflux, as well as accelerate positrons. Therefore it is critical to model the sheath field properly to obtain the correct yield and spectrum. The cylindrical geometry is required to accurately model the $1 / r^{2}$ fall-off of the E field, whereas the 2D Cartesian geometry would result in a $1 / r$ fall-off. The hot electrons were then re-sampled according to $\mathrm{f}(\mathrm{E}$, $\theta, \mathrm{r}, \mathrm{t}$ ) distribution and injected into a $1 \mathrm{~mm}$ thick, $1 \mathrm{~mm}$ diameter $\mathrm{Au}$ target in a $2 \mathrm{D}$ cylindrical geometry. Positron generation and transport was then simulated both inside and behind the Au target. To compare with the experimental results, statistics of escaped electrons and positrons were performed at another extraction plane that was $2 \mathrm{~mm}$ from the backside of the target.

The electron spectra generated from the LPI simulations are shown in Figure 3(c). The 
dashed curves are the raw distributions derived in Cartesian coordinates and the solid curves are converted distributions in cylindrical coordinates. The electron temperatures Te for different portions of the spectra are also labeled in the plot. After conversion, the temperature for higherenergy-range electrons is maintained at around $20 \mathrm{MeV}$, which is quite close to the experimentally measured temperature of $21 \mathrm{MeV}$. Lower energy electrons have a wider angular distribution and thus tend to be more easily affected by the conversion. Te decreased by about $3 \mathrm{MeV}$ for electrons within $25-70 \mathrm{MeV}$. Comparing optimal structure (red) to flat (black), the main difference appears at energies above $25 \mathrm{MeV}$, as the optimal structure tends to produce about an order of magnitude more electrons within this energy range.

To evaluate the positron yield, in Figure $3(\mathrm{~d})$ we have plotted $f(E) \cdot P_{e^{+}}(E)$, where $f(E)$ is the spectrum of injected electrons (solid curves in Figure 3(c)), and $P_{e^{+}}(E)$ is the probability that one positron could be generated and exit from the $1 \mathrm{~mm}$ thick, $1 \mathrm{~mm}$ diameter Au target as one incident electron with energy $\mathrm{E}$ is injected. $P_{e^{+}}(E)$ was obtained using a Monte Carlo code $\mathrm{MCNP}^{27}$ and the field effects have been ignored. The results are shown as the blue solid curve in Figure 3(d) on a log scale. The positron production probability grows sharply with energy for incident electrons below $\sim 30 \mathrm{MeV}$ and gradually saturates at high energies. The black and red solid curves indicate the calculated $f(E) \cdot P_{e^{+}}(E)$ for flat and optimally structured targets, respectively. Both curves peak at about $15 \mathrm{MeV}$. However, electrons within $25-150 \mathrm{MeV}$ from the optimal structure contributed to a great extent to the positron yield, whereas for the flat target most of the positrons are generated by lower energy electrons. Overall, the injection spectrum from an optimally structured target produced about $30 \%$ more positrons than the flat target. Note that this estimation does 
not consider any field or electron reflux effects that in reality play an important role. Higher energy electrons also tend to produce more forward going positrons. Assuming that all injected electrons have normal incidence, the dashed blue curve in Figure 3(d) shows the probability of one positron exit at $0^{\circ}$ from the backside of a Au target as one electron enters, i.e., positron per incident electron per sr at $0^{\circ}$. Multiplying this probability by the injection elelctron spectra yields the two dashed curves for flat (black) and optimally structured (red) targets, respectively. In this case, the peak contribution shifts to higher energies: about $27 \mathrm{MeV}$ for flat targets and $48 \mathrm{MeV}$ for the optimized structrue. Moreover, the optimal structure generates about twice as many forward going positrons at $0^{\circ}$ angle as the flat target.

The Monte Carlo simulation only provides an intuitive view of the pair production capability of LPI electrons. Understanding the energy difference in the measured positron spectra in contrast requires closer evaluation of the transport PIC simulations that involve the sheath field. The comparison of modeled and experimentally measured positron spectra at target normal (laser direction) is shown in Figure 4(a). The simulated spectra agree qualitatively with the experimental data. In Figure 4(b), the dark solid curves show the simulated spectra of escaped electrons at $0^{\circ}$ whereas for comparison the light solid curves in the background show the corresponding experimental spectra. Both spectra have a relatively good overlap within the energy range between $40 \mathrm{MeV}$ and $110 \mathrm{MeV}$. At lower energy, the mismatch is expected because the experimentally measured spectra include electrons that are generated at much later times than those covered by the simulation. The simulated spectra showed less particles at high energies. However, according to Figure 3(d), elelctrons above $110 \mathrm{MeV}$ would make a negligible contribution to the positron yield. These high 

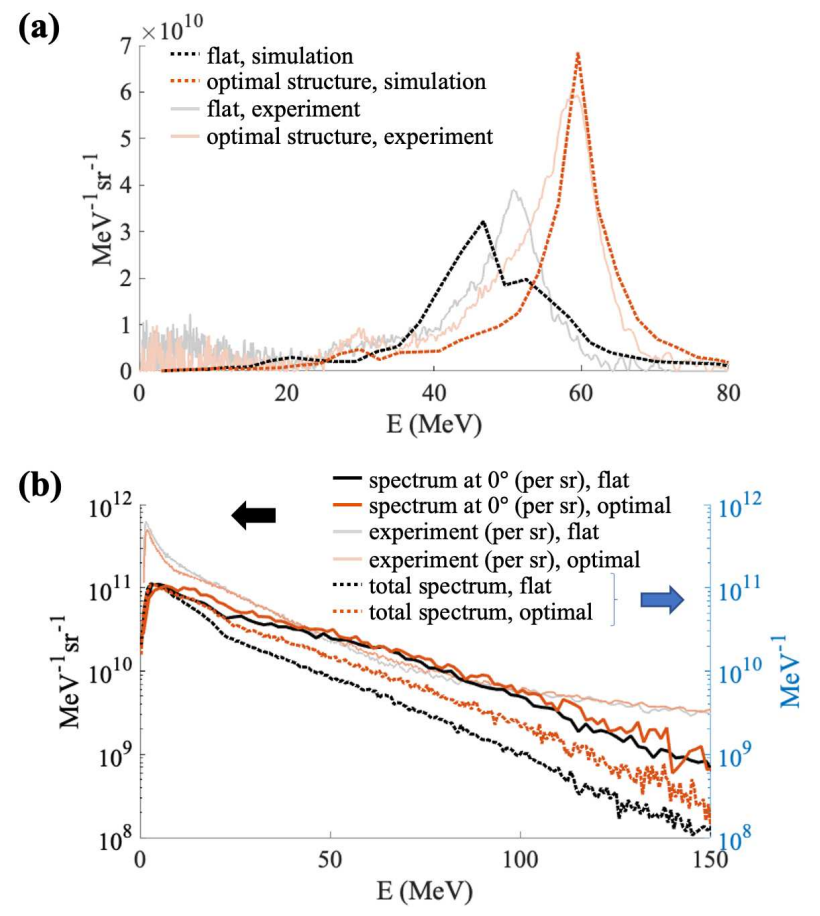

Figure 4: (a) Positron spectra at $0^{\circ}$ from simulations. (b) Electron spectra at $0^{\circ}$ (solid lines, with unit $\mathrm{MeV}^{-1} \mathrm{Sr}^{-1}$ on the left $\mathrm{y}$ axis) and overall electron spectra (dashed lines, with unit $\mathrm{MeV}^{-1}$ on the right $y$ axis). Note that the two different spectra plotted have mutually different units. We have also plotted corresponding experimental spectra at $0^{\circ}$ in the background for comparison. 
energy electrons have a small impact on the sheath field as well because their total charge is low. Therefore, the simulated positron and electron spectra indicate that the injected electron source from LPI simulation models the experimental condition reasonably well. For both the flat and the optimally structured target, the electron spectra measured at the target normal direction are mutually quite similar, whereas the positron spectra are obviously different, in accord with experimental observations. In Figure 4(b) we have also plotted the total electron spectrum (in $\mathrm{MeV}^{-1}$ ) as the dashed black and red curves. Unlike the spectra at $0^{\circ}$, the total spectrum from the optimally structured target clearly shows more high energy electrons, which explains the large discrepancy in the positron spectra, because forward going positrons are generated by all electrons, not just by the forward going ones.

The energy of positrons is largely determined by the sheath field on the back side of the target. Figure 5 shows the evolution of the sheath field $E_{z}$ as a function of the longitudinal position $\mathrm{z}$ and time t. Column (a) are the results from the flat target and column (b) are from the optimally structured target. Images (a1),(b1) and (a3),(b3) show the $E_{z}$ field at $\mathrm{r}=0$ and average $E_{z}$ field over the $1 \mathrm{~mm}$ diameter disk respectively, whereas (a2), (b2) and (a4), (b4) are the corresponding voltages $\mathrm{V}$ calculated by integrating $E_{z}$ over the longitudinal distance $\mathrm{z} . V=\int_{z_{o}}^{z} E_{z} d z$, where $z_{0}=$ $1 \mathrm{~mm}$ indicates the back surface of the target. These plots allow for an estimate of the accelerating capability of the sheath field. The images at $r=0$, indicate that passes of electrons gradually build up the sheath field on the target backside. Comparing the integrated voltage for flat and optimally structured targets, both the voltage at $r=0$ and the average voltage for the structured target are about $10 \mathrm{MV}$ higher than that for flat target, which is consistent with the measured 

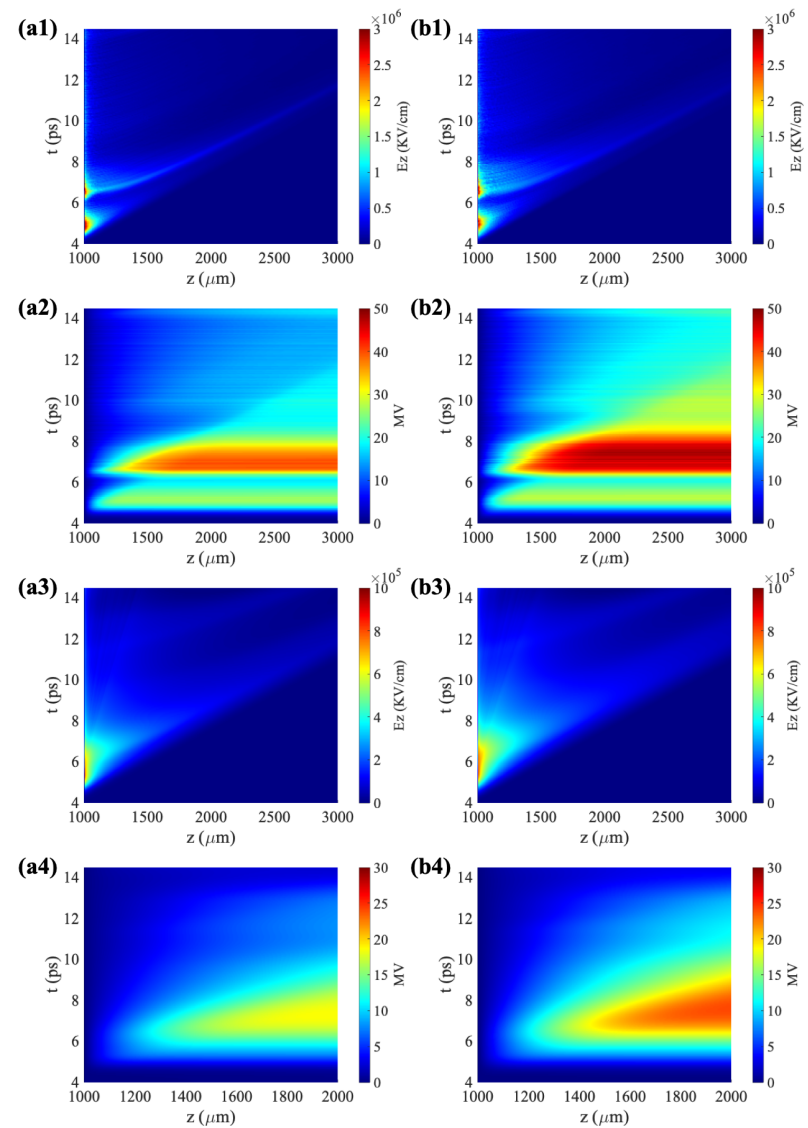

Figure 5: (a1), (b1) Sheath field $E_{z}$ at $\mathrm{r}=0$ as a function of time and longitudinal position z. (a2), (b2) corresponding voltage calculated by integrating $E_{z}$ over z. (a3), (b3) Average $E_{z}$ over the back surface of the target. (a4), (b4) corresponding voltage by integrating the average $E_{z}$. Here column (a) is for flat target and column (b) is for optimally structured target. 
energy difference between their positron peaks.

The two-stage PIC simulation successfully reproduced the experimental results, suggesting its potential for further target structure optimization to control the generation of positrons and other secondary particles, such as ions that are also greatly influenced by the sheath field. Optimal target parameters will vary substantially with laser pulse length, intensity, focal spot size, and the amount of prepulse. Nominally the wires need to be thick enough to survive the prepulse and the rising edge of the main pulse, but not too thick to interfere with laser propagation. Therefore, the desired diameter of wires increases with the laser pulse length and decreases with the laser contrast. The wire length needs to be longer than the direct laser acceleration length so that the electrons extracted from the wires by the laser can be accelerated to maximum speed. This acceleration length is determined by the laser intensity and the preplasma density. There is usually a generous range of wire lengths within which the wires would have a similar effect on the energy boost of electrons. The optimal spacing between wires is determined by the focal spot size as well as the scale of the preplasma. For high-energy, directional electron beam generation, the best result can be achieved when the micro-structure spacing is similar to the size of the focal spot and when a clean laser pulse is used. In contrast, reducing the structure spacing and introducing a proper amount of preplasma can enhance laser absorption. Therefore a compromise between the two effects has to be reached to maximize pair production.

The number of LPI electrons that are $10 \mathrm{~s}$ of $\mathrm{MeV}$ or higher determines the number of positrons that can be generated inside the convertor target as well as the charge that can escape 
from the target that subsequently determines the sheath field. For a given convertor target, e.g. $1 \mathrm{~mm} \mathrm{Au}$, the positron generation probability increases slowly for electrons above $\sim 40 \mathrm{MeV}$ according to Figure 3(d), and according to Figure 5, any electron that is above $\sim 50 \mathrm{MeV}$ can escape from the target. Therefore unlike previous work that used the structures for relativistic electron beam generation ${ }^{17,18}$, our goal here is not to accelerate the electrons to the highest possible energy, but to generate as many moderate energy (10s of $\mathrm{MeV}$ ) electrons as possible without sacrificing the laser conversion efficiency. Having a proper amount of preplasma is beneficial in our application. The current experiment was performed with a prepulse that naturally existed in the OMEGA EP laser. Future experiments with a controlled prepulse may further improve the positron yield. Another potential advantage of using the target structures is that the positron yield and energy are more sensitive to the laser intensity. The temperature Te of fast LPI electrons near the critical density can usually be estimated using the ponderomotive scaling $\mathrm{T}_{e} \approx 0.511 \times\left(\sqrt{1+I\left(W / \mathrm{cm}^{2}\right) \lambda^{2} / 1.4 \times 10^{18}}-1\right) \mathrm{MeV}^{28}$, and for high intensiy, Te approximately grows like the square root of the intensity. However, with the microwire array, the highest-energy electrons are accelerated via a different direct laser acceleration mechanism. The energy of this particular portion of the electrons scales linearly with laser intensity. Consequently, the positrons that are generated by them also tend to have a stronger dependence on intensity. Therefore the structured target would be more advantageous if higher-intensity lasers are developed in the future. Our PIC simulations have shown that even the "hot spot" in the laser focal spot cannot be ignored and is important to the resultant energy and number of positrons. 


\section{Conclusions}

In summary, front surface target structures have been shown experimentally to substantially enhance the positron yield and energy for the first time, constitiuting a cost-effective approach to use laser-generated positron sources for laboratory astrophysics applications. The follow-up simulations explain the entire process of how the laser-plasma interaction that is manipulated by the target structure affects the yield and energy of positrons. The agreement between the simulated and experimental spectra indicates the possibility of further target optimization using two-stage PIC simulations.

\section{References}

1. Piran, T. Rev. Mod. Phys. 76, 1143 (2005).

2. Chen, H. et al. High Energy Density Physics 7, 225 (2011).

3. Liang, E. et al. Sci. Report 5, 13968 (2015).

4. Sarri, G. et al. Nat. Comm. 6, 6747 (2015).

5. Stoneking, M., Pedersen, T., Helander, P. et al. A new frontier in laboratory physics: electronpositron plasmas. To be published.

6. Schwinger, J. Phys. Rev. 82, 664 (1951).

7. Breit, G. \& Wheeler, J. A. Phys. Rev. 46, 1087 (1934). 
8. Bell, A. R. \& Kirk, J. G. Phys. Rev. Lett. 101, 200403 (2008).

9. Sarri, G. et al. Phys. Rev. Lett. 110, 255002 (2003).

10. Chen, H. et al. Phys. Rev. Lett. 105, 015003 (2010).

11. Chen, H. et al. Phys. Plasmas 21, 040703 (2014).

12. Chen, H. et al. Phys. Rev. Lett. 114, 215001 (2015).

13. Xu, T. et al. Phys. Plasmas 23, 033109 (2016).

14. Williams, G. J. et al. Phys. Plasmas 23, 123109 (2016).

15. Yan, Y. et al. Plasma Phys. Control. Fusion 59, 045015 (2017).

16. Bethe, H. \& Heitler, W. Proc. Royal Soc. of London A 146, 83 (1934).

17. Jiang, S. et al. Phys. Rev. Lett. 116, 085002 (2016).

18. Jiang, S., Krygier, A. G., Schumacher, D. W., Akli, K. U. \& Freeman, R. R. Phys. Rev. E 89, 013106 (2014).

19. Jiang, S., Krygier, A. G., Schumacher, D. W., Akli, K. U. \& Freeman, R. R. Eur. Phys. J. D 68, 283 (2014).

20. Chicago is being developed by Voss Scientific with partial support from the Defense Advanced Research Projects Agency under Contract No. W31P4Q-15-C-0055 and the Air Force Office of Scientific Research under Contract No. FA9550-14-C-0034. 
21. Warren, E. L., Atwater, H. A. \& Lewis, N. S. J. Phys. Chem. C 118, 747 (2014).

22. Henry, M., Welch, C. \& Scherer, A. J. Vac. Sc. Technol. A 27, 1211 (2009).

23. Dorrer, C., Consentino, A., Irwin, D., Qiao, J. \& Zuegel, J. D. J. Opt. 17, 094007 (2015).

24. Marinak, M. M. et al. Phys. Plasmas 8 (2001).

25. Bartal, T. et al. nature phys. 8, 139 (2011).

26. Sawada, H. et al. Phys. Plasmas 19, 103108 (2012).

27. Los Alamos Scientific Laboratory. Group X-6. MCNP : a General Monte Carlo Code for Neutron and Photon Transport. Los Alamos, N.M. : [Springfield, Va.] :Dept. of Energy, Los Alamos Scientific Laboratory ; [for sale by the National Technical Information Service], 1979.

28. Wilks, S. C., Kruer, W. L., Tabak, M. \& Langdon, A. B. Phys. Rev. Lett. 69, 1383 (1992).

Acknowledgements We thank the OMEFA EP team for laser operation and technical support. This work was performed under the auspices of the U.S. DOE by LLNL under Contract DEAC5207NA27344, and funded by LDRD (\#17ERD010). The fabrication of Si microwire arrays was supported through the Office of Science of the U.S. Department of Energy under Award No. DE- SC0004993. Additional support for this work was provided by the Lockheed Martin Corporation (Award 4103810021). We thank the staff at the Kavli Nanoscience Institute at Caltech for their technical assistance with fabrication.

Competing Interests The authors declare that they have no competing financial interests. 
Correspondence Correspondence and requests for materials should be addressed to S. Jiang (email:

287 jiang8@1lnl.gov) or H. Chen (email: chen33@1lnl.gov). 


\section{Figures}

(a)

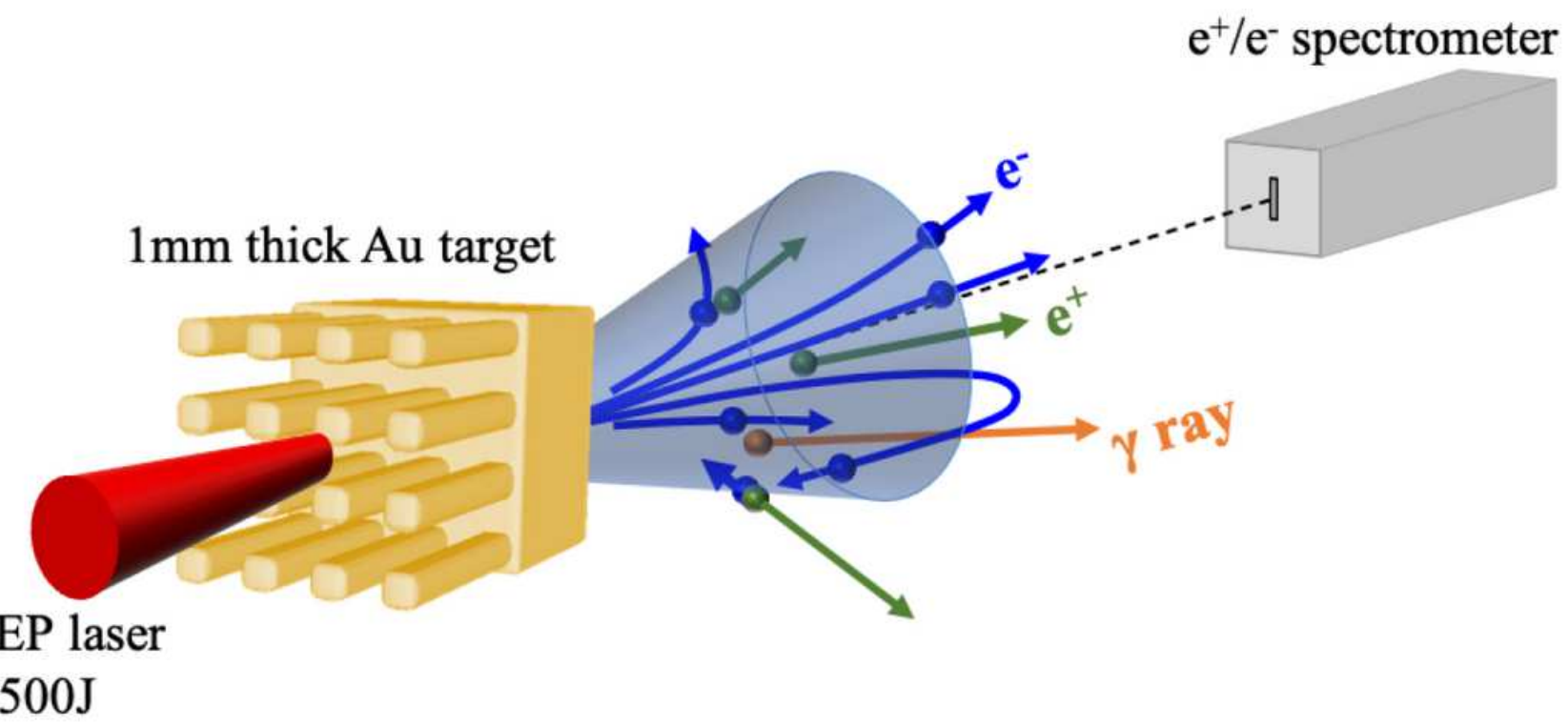

(b)

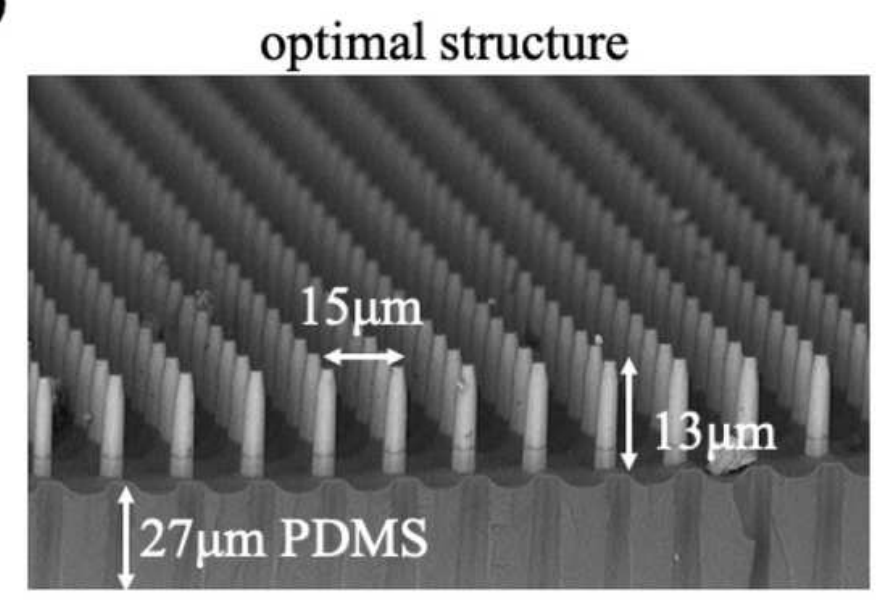

(c)

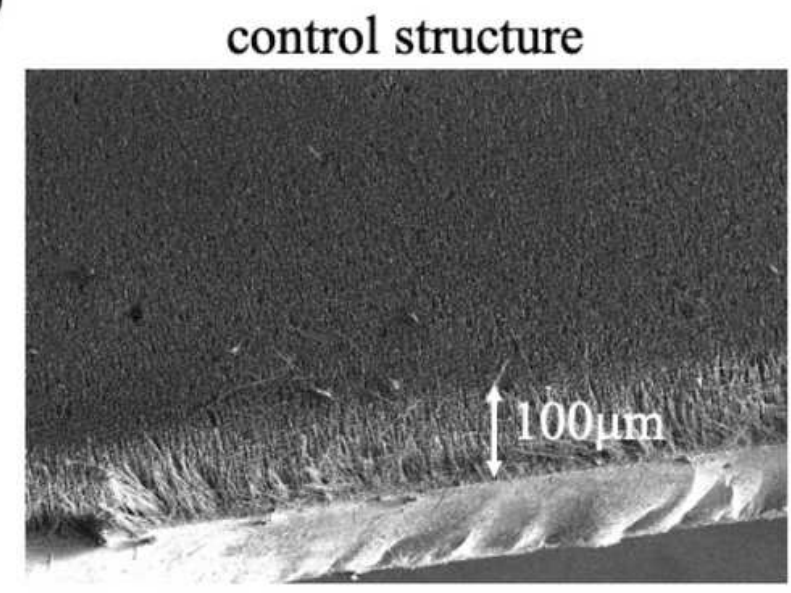

\section{Figure 1}

Schematic diagram of the experimental setup and scanning electron microscope (SEM) images of targets. (a) Schematic of the experimental setup. The same setup is used for LPI PIC simulations. The laser has $500 \mathrm{~J}$ energy, $700 \mathrm{fs}$ pulse length and a peak intensity of $4.46 \times 1020 \mathrm{~W} / \mathrm{cm} 2$. Target structures are made of Si wires that reside on a thin piece of polydimethylsiloxane. They are then attached to a $1 \mathrm{~mm}$ thick Au convertor target for positron generation. The electron/ positron spectrometer is placed opposite to the laser pulse. (b) SEM image of the optimized target structure. The wires are $3 \mu \mathrm{m}$ in diameter and $15 \mu \mathrm{m}$ apart. They have a total length of $40 \mu \mathrm{m}$ but their bottom parts are embedded inside a $27 \mu \mathrm{m}$ polydimethylsiloxane layer; therefore the wire structures exposed outside the polydimethylsiloxane is $13 \mu \mathrm{m}$. (c) SEM image of the unoptimized control structure used in the experiment. The Si wire array is about $100 \mu \mathrm{m}$ long (exposed outside polydimethylsiloxane), $3 \mu \mathrm{m}$ in diameter, and $7 \mu \mathrm{m}$ in period. 

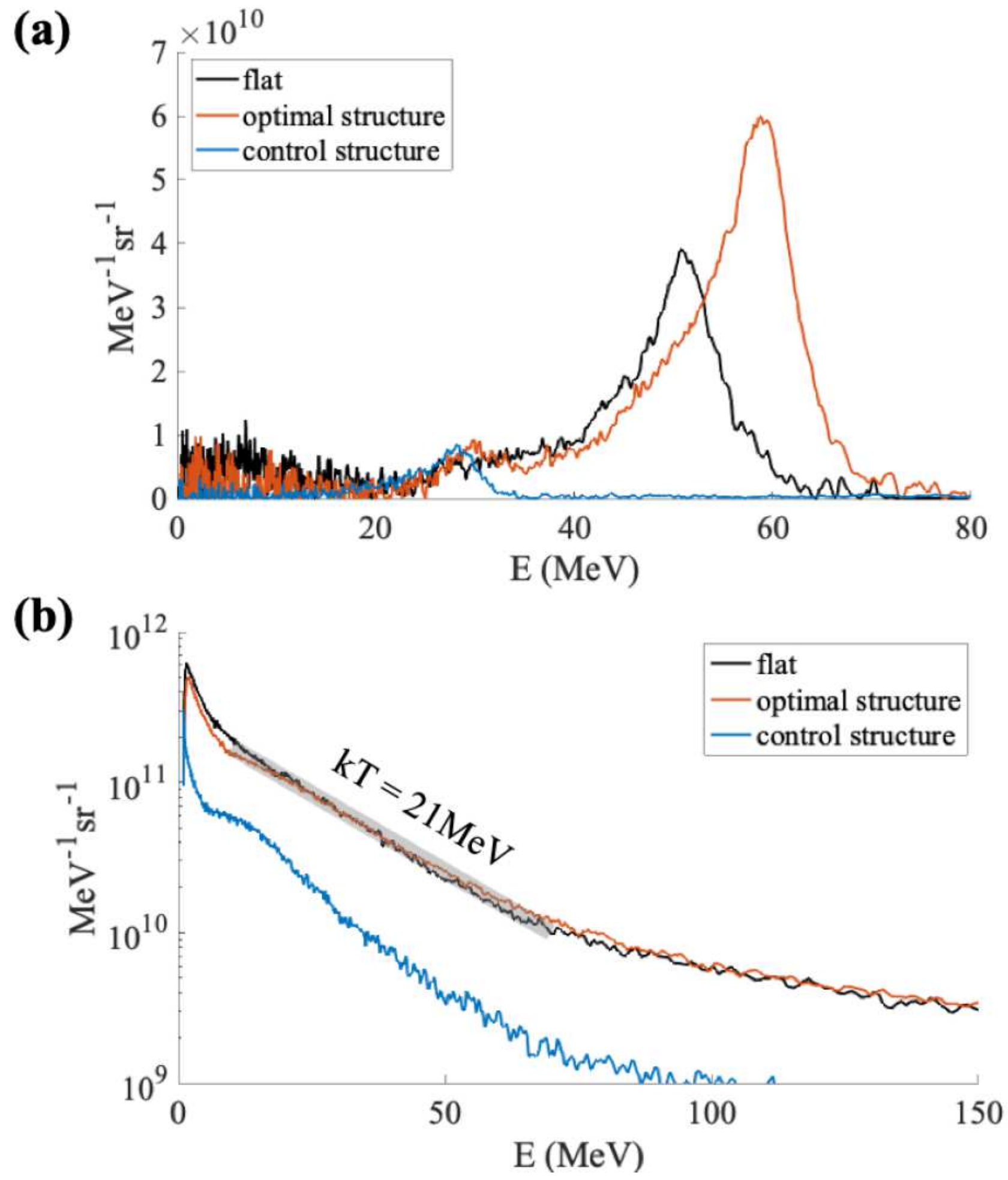

Figure 2

Experimentally measured spectra for (a) positrons and (b) electrons. Different colors indicate the results from different targets under the same laser conditions. 
(a)

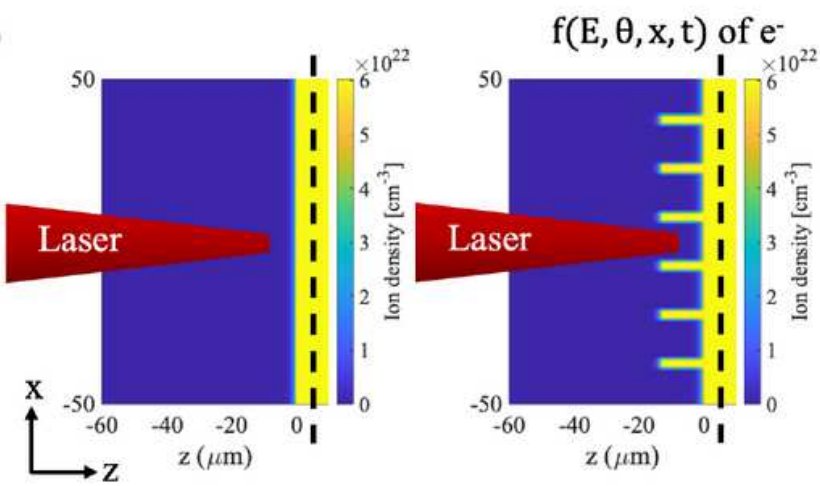

(c)

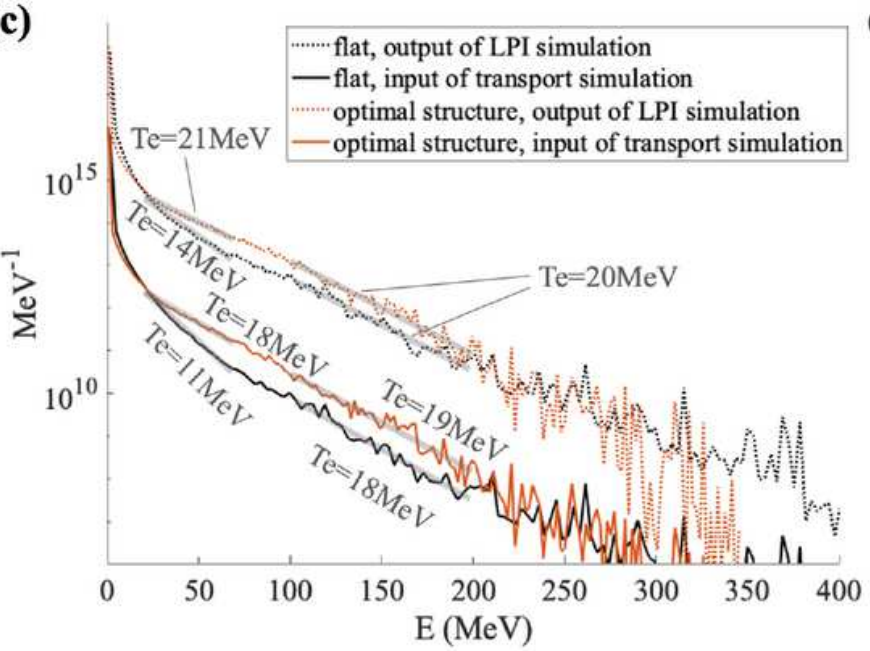

(b)

LPI simulation in $\quad \mathrm{e}^{-}$transport simulation

2D XZ coordinates in $2 \mathrm{D} \mathrm{RZ}$ coordinates

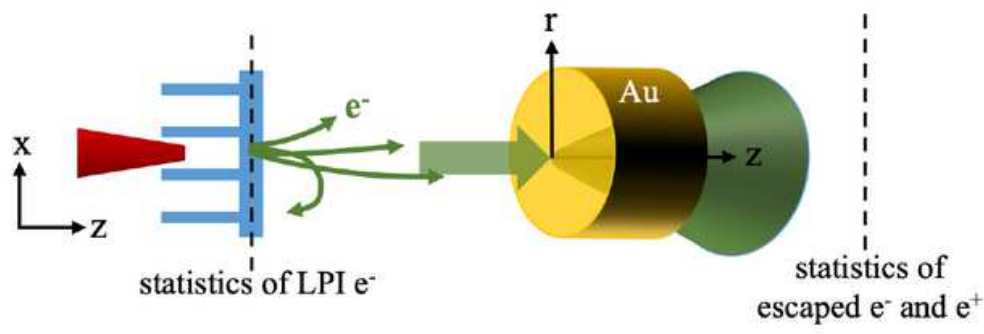

(d)

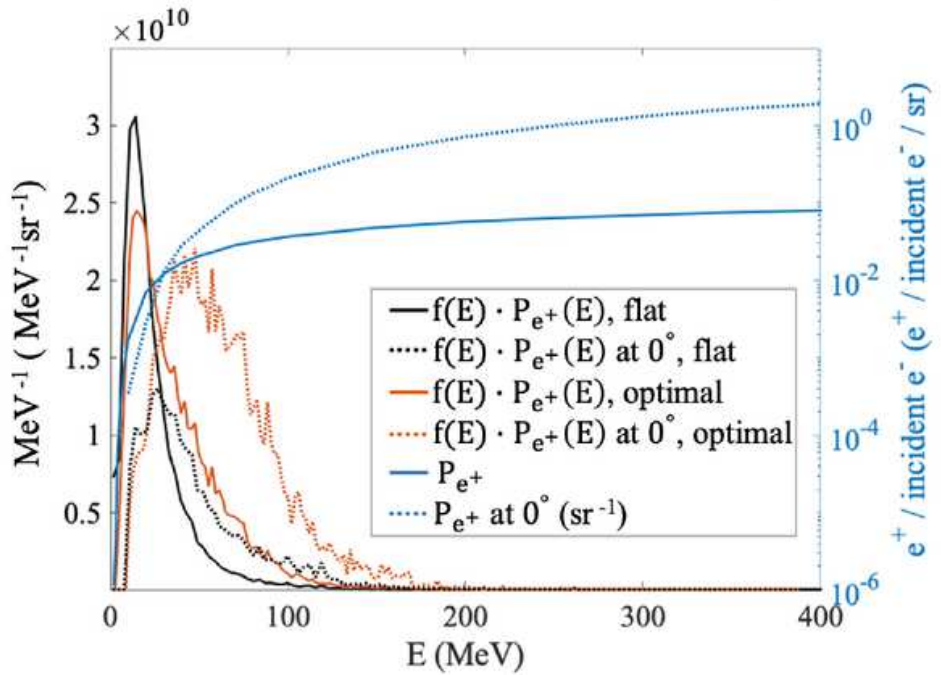

Figure 3

(a) Initial ion density for 2D Cartesian LPI simulations. (b) Schematic diagram of simulation setups. We have injected the fast electrons derived from LPI simulation to the following transport simulation after converting the electron source from Cartesian to cylindrical geometry. (c) Electron spectra inside the target from 2D Cartesian LPI simulations (dashed curves) and spectra of injected electron source for 2D cylindrical transport simulations (solid curves). (d)Solid blue curve (right y axis) shows the probability of one positron generated by one monoenergetic electron transporting through a $1 \mathrm{~mm}$ thick, $1 \mathrm{~mm}$ diameter Au target, and dashed blue curve shows the probability (per sr) of generating a positron that exits at $0^{\circ}$ with respect to target normal. The black and red curves (with respect to the left y axis) show injection electron spectra multiplied by the positron generation probability as a function of energy. 

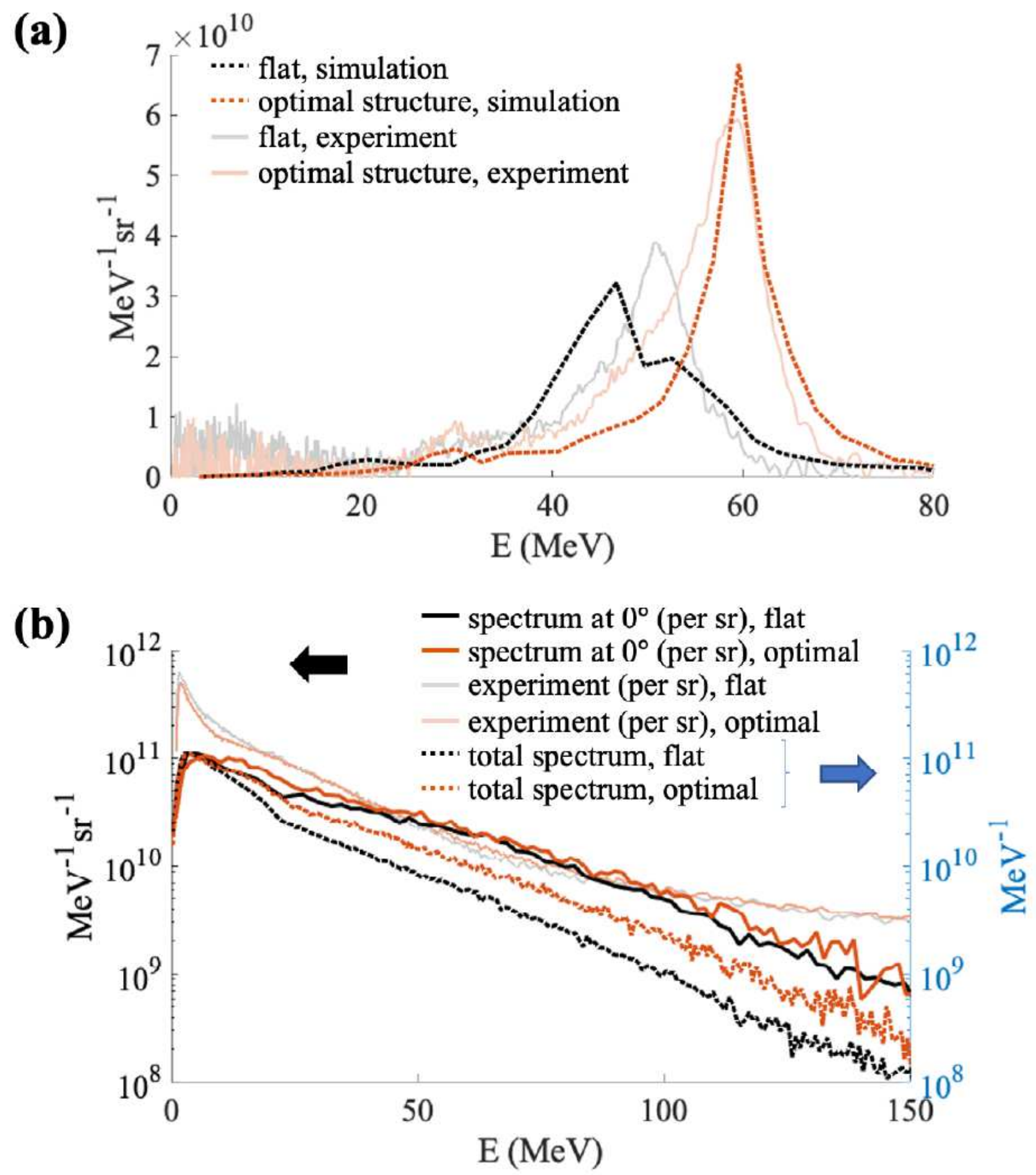

Figure 4

(a) Positron spectra at $0^{\circ}$ from simulations. (b) Electron spectra at $0^{\circ}$ (solid lines, with unit MeV- $1 \mathrm{sr}-1$ on the left $y$ axis) and overall electron spectra (dashed lines, with unit MeV-1 on the right y axis). Note that the two different spectra plotted have mutually different units. We have also plotted corresponding experimental spectra at $0^{\circ}$ in the background for comparison. 

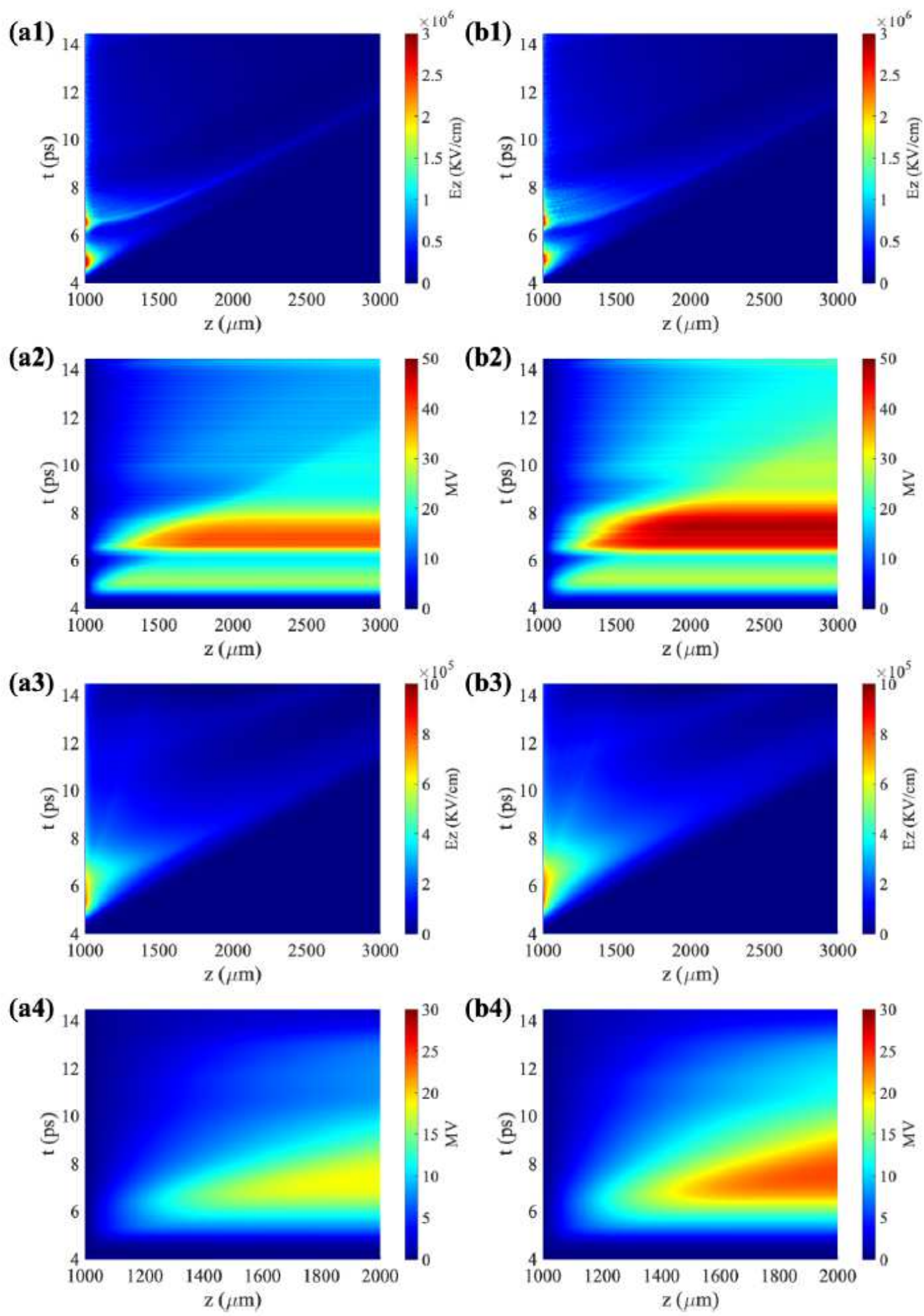

\section{Figure 5}

(a1), (b1) Sheath field Ez at $r=0$ as a function of time and longitudinal position z. (a2), (b2) corresponding voltage calculated by integrating Ez over z. (a3), (b3) Average Ez over the back surface of the target. (a4), (b4) corresponding voltage by integrating the average Ez. Here column (a) is for flat target and column (b) is for optimally structured target. 